\title{
Robótica Educacional como Facilitadora do Aprendizado do Raciocínio Computacional: Revisão Sistemática da Literatura
}

\author{
Ana Carolina Sokolonski ${ }^{1,2}$, Alirio Santos de Sá ${ }^{2}$, Raimundo José de Araújo Macêdo ${ }^{2}$ \\ ${ }^{1}$ Grupo de Pesquisa em Sistemas Embarcados, Controle e Eficiência Energética (GSECEE) \\ Departamento Acadêmico de Computação \\ Instituto Federal de Educação, Ciência e Tecnologia da Bahia (IFBA) \\ Rua Emídio dos Santos S/N - Salvador, BA - Brasil \\ ${ }^{2}$ Laboratório de Sistemas Distribuídos (LaSiD) \\ Programa de Pós-Graduação em Mecatrônica (PPGM) \\ Universidade Federal da Bahia (UFBA) \\ Av. Adhemar de Barros S/N - Salvador, BA - Brasil \\ carolsoko@ifba.edu.br, aliriosa@ufba.br, macedo@ufba.br
}

\begin{abstract}
Many educators and institutions have considered the Computational Thinking as essential for preparing future generations. As such, the development of Computational Thinking has been proposed and studied by means of distinct approaches, among them, the Educational Robotics (ER). ER uses basic robotics principles with the aim of developing skills not usually developed in schools. The ER can be an enabler of CT, because it uses an active learning methodology to encourage student learning through playful teaching. The purpose of this article is to assess the state of the art in the field of ER and CT through a systematic literature review involving all levels of education, considering the world scenario and using quantitative and qualitative metrics. Published works that investigate the potential of joint education were evaluated, presenting an analysis of how the ER has been used to foster students' CT and what are the open research fields.
\end{abstract}

Resumo. Muitos educadores e instituições têm considerado o Raciocínio Computacional ( $R C$ ) como essencial para o desenvolvimento do cidadão do futuro. Por Isso, o desenvolvimento do RC vem sendo proposto e estudado através de diversas abordagens, entre elas a utilização da Robótica Educacional (RE). A RE utiliza princípios básicos de robótica com o objetivo de desenvolver habilidades não desenvolvidas usualmente nas escolas. A RE pode ser potencializadora do RC, pois utiliza uma metodologia ativa de aprendizagem para incentivar o aprendizado dos alunos através de um ensino lúdico. A proposta deste artigo é avaliar o estado da arte da área de RE e RC através de uma Revisão Sistemática da Literatura envolvendo todos os níveis de ensino, considerando o cenário mundial e utilizando métricas quantitativas e qualitativas. Foram avaliados trabalhos publicados que investigam as potencialidades do ensino conjunto, apresentando uma análise acerca de como a RE tem sido utilizada para fomentar o $R C$ dos alunos e quais são os campos de pesquisa em aberto. 
IX Congresso Brasileiro de Informática na Educação (CBIE 2020)

Anais do XXXI Simpósio Brasileiro de Informática na Educação (SBIE 2020)

\section{Introdução}

Os fundamentos relativos ao raciocínio ou pensamento computacional, do Inglês "computational thinking", são antigos, notadamente a partir do conceito da máquina universal de Alan Turing. O termo Raciocínio Computacional (RC) ganhou notoriedade e maior interesse a partir da definição formulada por Jeannette Wing, em 2006 [Wing 2006], e aprimorada ao longo dos anos [Wing 2008, Wing and Reed 2010].

O RC consiste em uma maneira de organizar o raciocínio, podendo explorar habilidades de matemática, raciocínio lógico, resolução de problemas, abstração de problemas, entre outras. Jeannette Wing e outros autores defendem que qualquer pessoa pode e deve desenvolver e se apropriar desta forma de pensar, pois os benefícios de pensar como um cientista da computação vão além de saber como funciona um computador e não estão restritos aos problemas computacionais, lógica e matemática, abrangem todas as ações que realizamos em nosso cotidiano, relativas ao planejamento e medidas eficazes para resolver problemas e desafios [Wing 2008].

Para [Guzdial and Morrison 2016], o raciocínio computacional pode ser apresentado à criança na educação básica, propiciando seu pleno desenvolvimento do raciocínio lógico e plena expansão da capacidade de abstrair e expandir o pensamento humano como um todo. No momento em que se tem conhecimento dos efeitos esperados, pode-se definir o que é necessário para atingir os objetivos pretendidos e os métodos a serem adotados para alcançar essas metas, assim como o que não deve ser feito.

A Robótica Educacional (RE) surgiu a partir das teorias do Construtivismo de Jean Piaget e do Construcionismo de Seymour Papert. Em [Piaget 1976], Piaget defende que as crianças são seres pensantes, que constroem suas próprias estruturas cognitivas, aprendendo através da construção do seu próprio conhecimento, adquirindo-o à medida que vivencia suas experiências. Em [Papert 1980], Papert mostra caminhos para utilização dos computadores no ensino tradicional e adiciona à teoria do construtivismo a ideia de que o conhecimento pode ser obtido de forma mais efetiva caso a criança esteja engajada, de maneira consciente, na construção de algo tangível. Um dos princípios da teoria do Construcionismo é a criação de ambientes ativos de aprendizagem para que os alunos possam testar suas ideias. Em [Papert et al. 1986], Papert desenvolveu uma linguagem de programação totalmente voltada para a educação, o Logo, de fácil compreensão e manipulação, com alto poder de programação e que permite manipular um dispositivo robótico, sendo portanto o precursor da RE como é conhecida atualmente.

O desenvolvimento do RC através da RE pode trazer diversos benefícios ao aluno, além de estimular seu interesse em continuar aprendendo [Anwar et al. 2019]. À medida que os alunos desenvolvem os projetos robóticos, eles precisam analisar os problemas, planejar e desenvolver soluções, trabalhar em equipe, entrar em consenso sobre as melhores soluções, entre outras habilidades. O RC se destaca pela capacidade de resolução de problemas complexos através da abstração, decomposição e sistematização da solução, que leva a simplificação do problema, portanto o RC auxilia e faz parte da prática da RE [Avila et al. 2017].

A fim de investigar como a interação entre RC e RE vem sendo explorada nos diversos níveis de ensino, inclusive na formação de professores, quais são as áreas que ainda precisam de investigação mais aprofundada, quais as ferramentas mais utilizadas 
para o ensino de RE, entre outras vertentes, realizamos uma Revisão Sistemática da Literatura (RSL), que será apresentada neste artigo. A RSL realizada abrangeu um vasto escopo de busca, pesquisando nos maiores portais de periódicos nacionais e internacionais, considerando trabalhos realizados com alunos de todas as idades e níveis de ensino, além da formação docente.

Necessita-se ressaltar que, apesar do RC ser conhecido e trabalhado de diversas formas no ensino da computação, e de outras áreas, há bastante tempo, esta RSL se limitou a analisar trabalhos publicados a partir de 2006, marco em que o termo RC passou a ser difundido e usado na comunidade acadêmica. Ressalta-se também que foram analisados apenas os trabalhos que especificaram o uso da RE para desenvolver o RC, de forma explícita. Assim, foram encontrados 12421 trabalhos, selecionados os 121 mais relevantes, destes, 64 estudos empíricos.

Na Seção 2, serão apresentados os trabalhos relacionados. Na Seção 3, será apresentada a RSL realizada, a pergunta-chave, a estratégia de busca, a extração dos dados e a meta-análise dos dados, destacando as informações mais relevantes. Por fim, o artigo será finalizado no Seção 4, onde será apresentada a conclusão do trabalho.

\section{Trabalhos Relacionados}

Durante o processo de busca da presente Revisão Sistemática da Literatura (RSL), foram encontradas 12 RSLs correlatas, que agregaram boas contribuições para o estudo. Avaliando as RSLs de forma minuciosa, pode-se destacar suas diferenças em relação à presente revisão:

1. Em [Zanetti et al. 2016], os autores apresentam uma RSL dos trabalhos relacionados ao RC, sem avaliar sua interação com a RE. Foram avaliados os trabalhos publicados, em português, entre os anos de 2012 e 2015, focando em trabalhos desenvolvidos em 4 eventos científicos, analisando 16 artigos.

2. Em [Avila et al. 2017], os autores fizeram uma RSL analisando apenas 21 artigos, que utilizavam a RE como estratégia para o desenvolvimento de habilidades do RC. Os autores se restringiram a trabalhos com o ensino básico.

3. Em [Carvalho et al. 2017], os autores realizaram uma RSL sobre ensino de RC através de Objetos de Aprendizagem. A análise foi realizada sobre 39 artigos publicados nos repositórios digitais da Sociedade Brasileira de Computação (SBC), entre os anos de 2012 e 2017, abrangendo, em sua maioria, aplicativos e softwares, dando menos ênfase à RE.

4. Em [Souza et al. 2018], os autores investigaram os trabalhos que usavam Kit LEGO para ensinar RE, sem se aprofundar no desenvolvimento do RC.

5. Em [Istikomah and Budiyanto 2018], os autores fizeram uma RSL para avaliar a contribuição da RE e da abordagem construcionista no desenvolvimento do RC dos alunos. Avaliaram apenas 11 trabalhos que consideravam o uso de RE para o ensino do RC, dando foco principal a abordagem construcionista.

6. Em [Ioannou and Makridou 2018], os autores fizeram uma RSL para investigar o ensino conjunto de RE e RC, do jardim de infância ao ensino médio. Foram analisados apenas 9 estudos empíricos da área.

7. Em [Isnaini and Budiyanto 2018], os autores fizeram uma RSL nas bases de dados ScienceDirect, Springer Link e ACM Digital Library com o objetivo de encontrar 
trabalhos que listassem o que tem sido feito para desenvolver o RC usando RE em crianças da primeira infância.

8. Em [Werlich et al. 2018], os autores realizaram um mapeamento sistemático sobre o RC no Ensino Fundamental, com o intuito de verificar as metodologias e as estratégias didático-pedagógicas empregadas e as ferramentas utilizadas. Os autores avaliaram trabalhos publicados entre 2008 e 2018, realizando sua busca em apenas 4 fontes de pesquisa.

9. Em [Nascimento et al. 2018], os autores realizaram um mapeamento sistemático visando a investigação de como o RC, em conjunto com outras disciplinas, vem sendo ensinado no ensino básico. Os autores selecionaram 14 artigos, avaliando apenas um artigo que envolvia RE.

10. Em [Santos et al. 2018], os autores realizaram uma RSL sobre a utilização da RE no processo ensino-aprendizagem da disciplina Lógica de Programação. A pesquisa analisou o cenário brasileiro, avaliando 7 trabalhos publicados entre $2013 \mathrm{e}$ 2017.

11. Em [Jesus et al. 2019], os autores realizaram uma RSL para identificar como abordagens e métodos colaborativos de aprendizagem estão sendo empregados em atividades para o desenvolvimento do RC, não focando em RE. Os autores avaliaram 37 trabalhos, publicados entre 2014 e 2019.

12. Em [Anwar et al. 2019], os autores investigaram trabalhos publicados sobre RE. O RC foi investigado junto com as demais disciplinas de Ciências, Tecnologias, Engenharias e Matemática (STEM), não tendo sua avaliação individual realizada.

A RSL realizada neste trabalho abrangeu um escopo maior de busca, considerou trabalhos realizados em todos os níveis de ensino, inclusive na formação docente. Abrangeu o cenário nacional e internacional, avaliou trabalhos publicados nos últimos 14 anos. Foram encontrados 12421 trabalhos, selecionados os 121 mais relevantes. Desta forma, esta RSL difere das citadas anteriormente por ser mais ampla e completa, reunindo numa pesquisa todos os aspectos avaliados anteriormente, além de ser mais atual, por incluir em sua avaliação os trabalhos publicados nos últimos anos.

\section{Revisão Sistemática da Literatura (RSL)}

Revisão Sistemática da Literatura (RSL) é uma investigação com o objetivo específico de coletar trabalhos semelhantes de variadas fontes, tipos e autores, para que seja realizada uma análise estatística do tema [Higgins et al. 2019]. Para que uma pesquisa seja considerada RSL, as seguintes etapas devem ser realizadas: Pergunta de pesquisa, Estratégia de busca, Busca na literatura, Seleção dos estudos, Extração dos dados, Avaliação da qualidade metodológica dos estudos incluídos, Síntese dos dados, Avaliação da qualidade das evidências, Redação e publicação dos resultados.

Neste artigo, foi realizada uma RSL baseada nos guias [Kitchenham 2004] e [Okoli and Schabram 2010], para saber como o uso de RE vem sendo explorada para o desenvolvimento do RC nos alunos em todos os níveis de ensino, inclusive a formação docente.

\subsection{Pergunta-Chave e Estratégia de Busca}

O primeiro passo de uma RSL é especificar uma pergunta-chave que seja factível de ser respondida. Utilizando o acrônimo FINER, que determina que a pergunta-chave deve 
ser: Factível, Interessante, Nova (inovadora), Ética e Relevante, a seguinte pergunta foi determinada para a presente RSL: "Como a Robótica Educacional tem sido usada para desenvolver o Raciocínio Computacional?".

A partir desta pergunta, determinou-se as palavras chaves que deveriam ser pesquisadas: Robótica Educacional, Robótica, Raciocínio Computacional e Pensamento Computacional. Em seguida, focando nos resultados das buscas, foi-se especificada a STRING de busca da seguinte forma: (raciocínio computacional AND robótica) OR (pensamento computacional AND robótica). Em inglês, a STRING ficou da seguinte forma: (computational thinking AND robotics). A partir destas STRINGS de busca, foram realizadas pesquisas nas bases de dados, considerando dados atualizados até o dia 30 de abril de 2020, e os resultados podem ser observados na Tabela 1. Buscas em portais como o Google Scholar e Periódicos da CAPES abrangeram trabalhos publicados nos principais eventos nacionais, como Congresso Brasileiro de Informática na Educação (CBIE), Congresso Brasileiro de Educação (CBE) e Congresso da Sociedade Brasileira de Computação (CSBC), além de trabalhos publicados em portais específicos.

Tabela 1. Resultado da Busca nas Bases de Dados

\begin{tabular}{c|c|c}
\hline Base de Dados & URL & Qtde de Estudos Encontrados \\
\hline IEEEXplore & www.ieeexplore.com.br & 127 \\
\hline ScienceDirect & www.sciencedirect.com & 904 \\
\hline ACM Digital Library & www.dl.acm.org & 668 \\
\hline Periódicos da CAPES & periodicos.capes.gov.br & 3388 \\
\hline ERIC & eric.ed.gov & 24 \\
\hline Google Scholar & scholar.google.com & 6518 \\
\hline ResearchGate & www.researchgate.net & 792 \\
\hline Total de estudos & - & 12421 \\
\hline
\end{tabular}

A triagem dos estudos foi feita eliminando, primeiramente, os trabalhos duplicados e, em seguida, com base em títulos e resumos. Após ler o título dos trabalhos, foi possível eliminar trabalhos que não atendiam ao escopo da pesquisa. Um dos critérios de seleção empregado foi quanto ao idioma, selecionando artigos em português ou inglês. Foram eliminados trabalhos que não foram publicados em versão completa e os que não tinham a versão completa disponível para download.

Após esta etapa, iniciou-se a análise dos resumos. O objetivo era determinar se os trabalhos continham de fato contribuições para a pesquisa, sempre focando no objetivo da pesquisa, explicitado pela pergunta-chave, e descartando trabalhos que abordassem superficialmente o RC ou a RE. Respeitando a abordagem conservadora e preservando o máximo de trabalhos possíveis, foram selecionados 121 trabalhos $^{1}$, que foram lidos em sua íntegra e serão avaliados na Subseção 3.2. Os critérios de elegibilidade utilizados se referem a estudos que avaliem a $\mathrm{RE}$ em conjunto com o RC, mesmo que não foque em suas habilidades.

\subsection{Extração de Dados dos Artigos Elegíveis e Meta-Análise}

Os artigos selecionados e analisados foram publicados entre os anos de 2006 e 2020. Pode-se observar sua distribuição temporal através do gráfico da Figura 1. A maioria

\footnotetext{
${ }^{1}$ http://www.lasid.ufba.br/publicacoes/artigos/FontesRSL-SBIE-2020.pdf
} 
dos trabalhos foram publicados nos anos de 2017, 2018 e 2019, o que mostra o quanto o tema desta RSL é atual. A queda na quantidade de publicações no ano de 2020 não é relevante, pois a pesquisa nas bases de dados foi realizada até o dia 30 de abril de 2020, não incluindo todas as publicações ocorridas no ano.

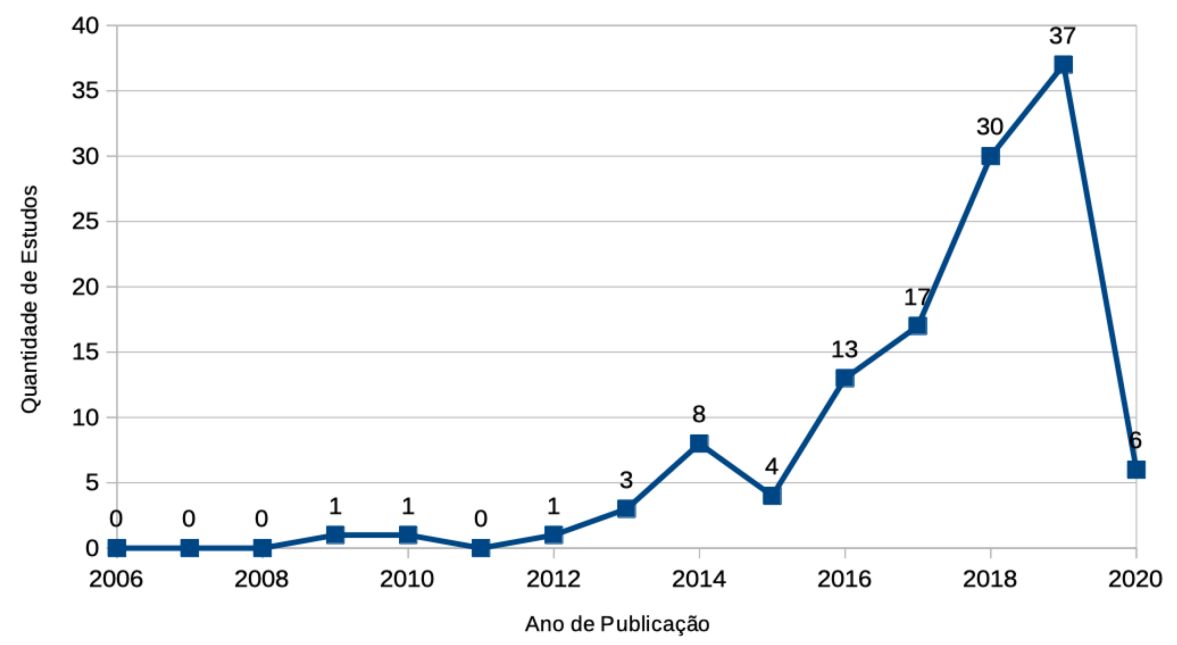

Figura 1. Quantidade de Estudos Selecionados X Ano de Publicação

A partir dos trabalhos selecionados, pode-se perceber que o RC vem sendo investigado em conjunto com a RE, inclusive, alguns trabalhos sugerem e experimentam o uso da RE como estimuladora do aprendizado do RC. A síntese dos dados pode ser observada na Tabela 2, onde estes foram classificados pelo tipo de estudo realizado.

Tabela 2. Tipos de Estudos Analisados na RSL

\begin{tabular}{c|c|c}
\hline Tipo do Estudo & Quantidade de estudos & Percentual do total \\
\hline Estudos Empíricos & 64 & $52.89 \%$ \\
Revisão Sistemática da Literatura & 12 & $9.92 \%$ \\
Demais Revisões & 5 & $4.13 \%$ \\
Propostas de Abordagens & 37 & $30.58 \%$ \\
Discussões e Análises & 3 & $2.48 \%$ \\
\hline Total & 121 & $100 \%$ \\
\hline
\end{tabular}

Percebe-se que, a maioria dos estudos encontrados são estudos empíricos ou propostas de abordagem. Foram encontradas 12 RSLs sobre temas correlatos, o que representa 9,92\% dos trabalhos da área. Estas RSLs foram apresentadas na Seção 2.

Pode-se perceber que $85,94 \%$ dos estudos empíricos trabalham com alunos de até 17 anos, sendo o ensino fundamental e médio o principal alvo dos pesquisadores, como pode ser visto na Tabela 3. Ou seja, a sugestão do ensino do RC em conjunto com a RE desde o ensino básico vem sendo bem aceita pela comunidade acadêmica, porém ainda é pouco praticada na maioria dos países relatados nestes estudos [Isnaini and Budiyanto 2018].

Dos 64 estudos empíricos encontrados, 23 investigavam a primeira infância, como pode ser visto na Tabela 3, ou seja, 35,94\%. Quantitativamente, 23 estudos, em um cenário mundial, é uma quantidade insatisfatória e não atende à necessidade de investigação 
de como inserir RC, usando como abordagem a RE, desde os primeiros anos escolares das crianças.

O somatório dos estudos especificados na Tabela 3 ultrapassa 100\%, pois alguns estudos avaliaram mais de uma faixa etária.

Tabela 3. Faixa Etária dos Alunos Investigados Nos Estudos Empíricos

\begin{tabular}{c|c|c}
\hline Idades dos Alunos & Quantidade de artigos & Percentual do total \\
\hline Idade até 11 anos & 23 & $35.94 \%$ \\
Idade de 12 a 16 anos & 32 & $50.00 \%$ \\
Idade de 17 a 18 anos & 11 & $17.19 \%$ \\
Idade acima de 18 anos & 5 & $6.15 \%$ \\
\hline Total & 64 & $100 \%$ \\
\hline
\end{tabular}

Outro aspecto observado nos estudos foi o uso de kits de RE disponíveis no mercado, poucos foram os trabalhos que desenvolveram o próprio kit customizado às necessidades de seus estudantes, porém, ainda assim, o entregaram montados aos mesmos.

Dos estudos que especificaram a abordagem robótica utilizada, 24 usaram Kit $L E G O, 14$ usaram outros kits disponíveis no mercado e 10 desenvolveram os próprios kits, como pode ser observado na Tabela 4. Portanto, 48 estudos, 39,67\%, usaram kits de fácil montagem. Apenas 20 estudos, $16,53 \%$ do total, optaram por deixar os alunos construírem o próprio dispositivo, a partir de elementos básicos.

O uso de kits de RE montados é indicado quando os alunos, além de não possuírem conhecimentos para a construção do dispositivo, não possuem maturidade para absorvê-los ao longo do processo, como é o caso dos alunos da primeira infância [Isnaini and Budiyanto 2018]. Trabalhos desenvolvidos com alunos no ensino médio e ensino superior poderiam ser realizados através da construção dos equipamentos, usando dispositivos eletrônicos básicos, para que o aluno pudesse desenvolver habilidades de RE e absorver todo o conhecimento associado [Junior et al. 2019].

Tabela 4. Recursos de Robótica Utilizados

\begin{tabular}{c|c|c}
\hline Recursos de robótica & Quantidade de artigos & Percentual do total \\
\hline Kit LEGO & 24 & $19.83 \%$ \\
Kits disponíveis no mercado & 14 & $11.57 \%$ \\
Kits desenvolvidos pelo autor & 10 & $8.26 \%$ \\
Arduino ou RaspberryPI & 20 & $16.53 \%$ \\
Simulação Virtual & 6 & $4.96 \%$ \\
Não especificou a abordagem & 47 & $38.84 \%$ \\
\hline Total de estudos encontrados & 121 & $100 \%$ \\
\hline
\end{tabular}

Um dos maiores obstáculos para a implantação da abordagem de ensino de RC através da RE nas escolas é a formação dos professores [Ramos and Espadeiro 2014]. Esta proposta de ensino vem sendo investigada e introduzida no currículo escolar nos últimos anos, porém ainda não faz parte da realidade dos cursos de formação docente na maioria dos países, necessitando de pesquisas que investiguem como viabilizar tal formação [Kousis 2019]. 
Ao considerar o cenário brasileiro, na última revisão da Base Nacional Comum Curricular (BNCC), foi introduzido o ensino de RC na educação básica como elemento obrigatório, não especificando a abordagem de ensino a ser utilizada [Brasil 2020]. A BNCC incluiu em uma das dez competências do aluno da educação básica:

Compreender, utilizar e criar tecnologias digitais de informação e comunicação de forma crítica, significativa, reflexiva e ética nas diversas práticas sociais (incluindo as escolares) para se comunicar, acessar e disseminar informações, produzir conhecimentos, resolver problemas e exercer protagonismo e autoria na vida pessoal e coletiva. [Brasil 2020, pag 9]

Portanto, faz-se necessário preparar os educadores de forma adequada. Como introduzir o RC no currículo deve ser alvo de pesquisa, tendo em mente que o tempo de aprendizado das crianças pode ser utilizado com propriedade, consciência e planejamento, visando o desenvolvimento pleno de seu potencial. Apesar destas considerações, apenas 14 estudos dos 121 analisados, 11,57\%, envolviam a formação docente, apresentando propostas de abordagens ou estudos empíricos.

Tabela 5. Habilidades do Raciocínio Computacional Investigadas nos Estudos Empíricos

\begin{tabular}{c|c|c}
\hline Habilidade & Quantidade de trabalhos & Percentual dos estudos empíricos \\
\hline Abstração & 12 & $18.75 \%$ \\
Decomposição & 10 & $15.63 \%$ \\
Generalização & 9 & $14.06 \%$ \\
Raciocínio Algorítmico & 16 & $25.00 \%$ \\
Avaliação & 8 & $12.50 \%$ \\
Demais habilidades & 13 & $20.31 \%$ \\
\hline Total de artigos & 19 & $29.69 \%$ \\
\hline
\end{tabular}

As habilidades do RC mais investigadas nos estudos empíricos são a abstração, decomposição, generalização, avaliação e o raciocínio algorítmico (sequenciação). Apenas 19 trabalhos especificaram as habilidades do RC investigadas, o que representa 29,69\% do total de estudos empíricos, como pode ser visto na Tabela 5. Uma das habilidades mais importantes, a abstração, foi investigada em apenas 18,75\% dos trabalhos. O raciocínio algorítmico foi citado em alguns trabalhos devido a necessidade do desenvolvimento de algoritmos para a manipulação de robôs, mas não teve sua evolução investigada. Muitos trabalhos descrevem que houve evolução do RC dos alunos, porém não avaliam as habilidades desenvolvidas e seus impactos.

\section{Conclusão}

A partir das análises realizadas, pode-se observar que existem duas áreas de pesquisa carentes de aprofundamento. A primeira consiste em avaliar as habilidades do Raciocínio Computacional (RC) que são desenvolvidas quando este é ensinado a partir da Robótica Educacional (RE), pois, apesar de 29,69\% dos estudos empíricos terem abordado o ensino/aprendizagem de habilidades do RC, observa-se que apenas 15,63\% avaliaram a decomposição, por exemplo. Desta forma, nota-se a necessidade de trabalhos que avaliem todas as habilidades do RC e as relacione adequadamente com o uso da RE no processo 
de ensino. A segunda consiste em pesquisas com o foco na formação docente, tornandoa mais adequada às necessidades do ensino de RC e RE. Desde a última atualização da BNCC, RC passou a ser uma das dez competências do ensino básico, obrigando os educadores a rever o currículo da educação básica e adequar sua formação às novas exigências.

Portanto, após as análises realizadas nesta RSL, pode-se concluir que a RE vem sendo investigada para estimular o aprendizado do RC de diversas formas diferentes. Porém, para um melhor planejamento e desenvolvimento de atividades direcionadas para $\mathrm{o}$ aprimoramento do RC dos alunos, necessita-se saber quais são as habilidades do RC que são desenvolvidas durante o ensino da RE, além de contar com professores capacitados para conduzir os alunos ao aprendizado do RC.

\section{Referências}

Anwar, S., Bascou, N. A., Menekse, M., and Kardgar, A. (2019). A systematic review of studies on educational robotics. Journal of Pre-College Engineering Education Research (J-PEER).

Avila, C., Cavalheiro, S., Bordini, A., and Marques, M. (2017). O pensamento computacional por meio da robótica no ensino básico - uma revisão sistemática. In Simpósio Brasileiro de Informática na Educação - SBIE, volume 28, page 82.

Brasil (2020). Base Nacional Comum Curricular (BNCC). Ministério da Educação. http://basenacionalcomum.mec.gov.br/.

Carvalho, J., Netto, J. F., and Almeida, T. (2017). Revisao sistemática de literatura sobre pensamento computacional por meio de objetos de aprendizagem. In Simpósio Brasileiro de Informática na Educação - SBIE, volume 28, page 223.

Guzdial, M. and Morrison, B. (2016). Growing computer science education into a stem education discipline. Communications ACM, 59(11):31-33.

Higgins, J., Thomas, J., Chandler, J., Cumpston, M., Li, T., Page, M., and Welch, V. (2019). Cochrane Handbook for Systematic Reviews of Interventions. John Wiley \& Sons, Chichester (UK), 2nd edition.

Ioannou, A. and Makridou, E. (2018). Exploring the potentials of educational robotics in the development of computational thinking: A summary of current research and practical proposal for future work. Springer Science - Education and Information Technologies, 23.

Isnaini, R. and Budiyanto, C. (2018). The influence of educational robotics to computational thinking skill in early childhood education. The 1st International Conference on Computer Science and Engineering.

Istikomah, I. and Budiyanto, C. (2018). The contribution of educational robotics and constructivist approach to computational thinking in the 21st century. The 1st International Conference on Computer Science and Engineering.

Jesus, A. M., Silveira, I. F., and de Lima Palanch, W. B. (2019). Desenvolvimento do pensamento computacional por meio da colaboração: uma revisão sistemática da literatura. Revista Brasileira de Informática na Educação - RBIE, 27:69. 
IX Congresso Brasileiro de Informática na Educação (CBIE 2020)

Anais do XXXI Simpósio Brasileiro de Informática na Educação (SBIE 2020)

Junior, A. d. O., Nascimento, L. T., and Macedo, D. (2019). Licenciatura em computação: Um relato de experiência utilizando robótica na formação de professores. In Anais do Congresso Brasileiro de Informática na Educação - CBIE, volume 8, page 71.

Kitchenham, B. (2004). Procedures for performing systematic reviews. Technical report, Keele University - UK.

Kousis, A. (2019). The impact of educational robotics on teachers' computational thinking. Educational Journal of the University of Patras UNESCO Chair, pages 426-432.

Nascimento, C., Santos, D. A., and Tanzi, A. (2018). Pensamento computacional e interdisciplinaridade na educação básica: um mapeamento sistemático. In Anais do Congresso Brasileiro de Informática na Educação - CBIE, volume 7, page 709.

Okoli, C. and Schabram, K. (2010). A Guide to Conducting a Systematic Literature Review of Information Systems Research. Working Papers on Information Systems, 10(26): $1-51$.

Papert, S. (1980). Mindstorms: Children, Computers, and Powerful Ideas. Basic Books, Inc., USA.

Papert, S., Valente, J., and Bitelman, B. (1986). Logo: computadores e educação. Brasiliense.

Piaget, J. (1976). Piaget and His School: A Reader in Developmental Psychology. Springer Berlin Heidelberg, Berlin, Heidelberg.

Ramos, J. L. and Espadeiro, R. G. (2014). Os futuros professores e os professores do futuro. os desafios da introdução ao pensamento computacional na escola, no currículo e na aprendizagem. Revista Educação, Formação e Tecnologias, 7:4-25.

Santos, F. E., Pereira, D. S., Godin, J. M., De Lima, J. V., Zaro, M. A., and do Canto Filho, A. B. (2018). A robótica educativa no ensino de lógica de programação: uma revisão sistemática da literatura. RENOTE - Revista Novas Tecnologias na Educação, 16.

Souza, I., Andrade, W., Sampaio, L., Araujo, A. L., and Araujo, S. (2018). A systematic review on the use of lego robotics in education. 2018 IEEE Frontiers in Education Conference (FIE).

Werlich, C., Kemczinski, A., and Gasparini, I. (2018). Pensamento computacional no ensino fundamental: um mapeamento sistemático. In XXIII Congresso Internacional de Informática Educativa, pages 375-384.

Wing, J. (2006). Computational thinking. Communications of the ACM, 49:33-35.

Wing, J. (2008). Computational thinking and thinking about computing. Philosophical transactions Series A: Mathematical, physical, and engineering sciences, 366:37173725 .

Wing, J. and Reed, D. (2010). Nsf funding advice: 21st century innovation. Communications of the ACM, 53:10-11.

Zanetti, H., Borges, M., and Ricarte, I. (2016). Pensamento computacional no ensino de programação: Uma revisão sistemática da literatura brasileira. In Simpósio Brasileiro de Informática na Educação - SBIE, volume 27, page 21. 